\title{
Experimental Analyses for The Mechanical Behavior of Pressed All- Ceramic Molar Crowns with Anatomical Design
}

\author{
Liliana Porojan ${ }^{1}$, Sorin Porojan ${ }^{2}$, Lucian Rusu ${ }^{3}$, Adrian Boloș ${ }^{2}$ and Cristina Savencu ${ }^{1}$ \\ ${ }^{1}$ Department of Dental Prostheses Technology, School of Dentistry, V. Babeş University of Medicine and Pharmacy, 9 Revolutiei 1989 Blv., \\ 300041 Timişoara, Romania \\ ${ }^{2}$ Department of Oral Rehabilitation, School of Dentistry, V. Babeş University of Medicine and Pharmacy, 9 Revolutiei 1989 Blv., 300041 \\ Timişoara, Romania \\ ${ }^{3}$ Department Mechanics and Vibrations, Faculty of Mechanical Engineering, Politehnica University, 1 Mihai Viteazu Street, 300222 \\ Timisoara, Romania
}

\begin{abstract}
Ceramic restorations show considerable variation in strength and structural reliability regarding to the type of material, and design characteristics. The fracture of ceramics occurs with little or no plastic deformation, with cracks propagated in an unstable manner under applied tensile stresses. The aim of the study was to assess experimental analyses of pressed monolithic ceramic crowns with anatomical design used in the posterior areas in order to understand their mechanical behavior before following their clinical use. Experiments were conducted on a complete molar crown preparation. Experiments show different modes of fracture for the tested samples. Digital images from the fractured pieces of the crowns were used to verify the fragments in all cases final fracture occurred by splitting into two and often more parts. The graphically representation of the displacement depending on the load highlights a series of peaks that can be correlated with cracks occurred in crowns. The development of well-designed mechanical experiments could be useful to help to predict clinical survival of these new all-ceramic restorative techniques and materials. Because failure is often accompanied by complete cracking of the crowns, preliminary research should represents a compulsory goal.
\end{abstract}

\section{Introduction}

Despite progress of ceramic materials and their technologies made in the last decade, all-ceramic restorations still have lower clinical longevity compared to metal-ceramic restorations, due to their brittle nature [1]. All-ceramic restorations are amongst the most aesthetically prosthetic restorations because they exhibit life-like appearance due to the lack of metallic substructure.

The fracture of ceramics occurs with little or no plastic deformation, with cracks propagated in an unstable manner under applied tensile stresses. Ceramic restorations show considerable variation in strength and structural reliability regarding to the type of material, and design characteristics $[2,3]$.

Different microstructures, depending on the presence and volume of the crystalline phase, mean particle size and distribution throughout the glassy matrix affect crack propagation and also the mechanical behavior [4]. It is also important to mention that other factors such the thickness and shape of restoration, flaws inserted during the manufacturing and the presence of residual stresses can also influence clinical longevity, making a direct correlation between the parameters of crack growth and an estimate of lifetime for a ceramic restoration more difficult [5].

Due to the complex geometry of the tooth crown and contact during mastication, fracture evaluation of ceramic all-ceramic crowns have often been simplified in different studies as a flat layered structure. Other studies considered the influence of surface curvature [6,7].

Recently monolithic all-ceramic crowns are increasingly favored over traditional bilayered crowns, which are associated with complex fabrication, presence of residual stresses, and chipping of the veneers [8-11].

Sintered feldspathic and leucite reinforced ceramic are used for the fabrication of metal-free restorations using traditional laboratory based fabrication techniques. With the development of hot-pressed and CAD/CAM technologies, different ceramic materials can now be used for aesthetic applications.

Ceramics are brittle and susceptible to fatigue fracture in repetitive function. Although occlusal loading is compressive, some tensile stresses in the restorations are inevitable. Cracks tend to follow paths where tensile stresses are greatest. Fracture during static load differ from those resulted after an extended period of lower loads $[12,13]$.

Even their many advantages, glass ceramics are brittle materials. Crack initiation and propagation can result in 
compromise of the restorations. The compromised structural integrity indicated by a significant reduction in fracture strength will be reflected in a poor clinical performance. Therefore the clinical significance of these results is to give an indication of the strength of hotpressed ceramic crowns to be used in the molar areas as monolithic restorations.

The aim of the study was to assess experimental analyses of pressed monolithic ceramic crowns with anatomical design used in the posterior areas in order to understand their mechanical behavior before their clinical use.

\section{Materials and method}

Experiments were conducted on a complete crown preparation made on a first maxillary molar from a typodont model. The axial undercuts were eliminated by a reduction of $1.5 \mathrm{~mm}$, while the occlusal surface was reduced by $1.5-2 \mathrm{~mm}$. A $1 \mathrm{~mm}$ deep chamfer finish line and a convergence angle of 6 degree were chosen. Finally all sharp angles were rounded.

This preparation was replicated and alloy dies were obtained by casting, using the lost-wax technique. The dies were scanned using Cercon Eye system and a dedicated CAD (computer aided design) software Cercon Art (Degudent, Hanau, Germany) was used for the design of the full anatomically crowns.

In order to obtain identical samples, eight wax-ups were milled in Cercon base cast material. These were invested, and specific steps were covered according to the manufacturer's instructions in order to obtain heat pressed ceramic crowns using Cergo pressed ceramics (Degudent, Hanau, Germany). The restorations thickness was verified by periodic measurements at several locations. The crowns were glazed and conventionally cemented on the metallic dies.

A digital compressive load was applied to the occlusal surface of each crown during setting to ensure complete seating and maintained for 24 hours before loading. The specimens were mounted into an Instron 8874 (Instron, Norwood, MA, USA) testing device and subjected to failure testing by compressive loading.

Each crown was positioned under a stainless steel ball with a diameter of $6 \mathrm{~mm}$ fixed to the upper crosshead of a universal testing machine. Due to the radius of the contact sphere, the actual contact positions were located adjacent to the central fossa. A thin rubber foil of $0.2 \mathrm{~mm}$ was inserted between the tested crowns and the antagonists in order to reduce peak stresses at the contact points.

A compressive load was applied at a crosshead speed of $2 \mathrm{~mm} / \mathrm{min}$, and failure was recorded. The vertical load was aligned with the tooth's axis.

The initial crack observed in the monolithic materials occurred at the same time as the ultimate failure of the crown, so the crack origin could not be exactly determined visually, but in all cases it started from the contact area with the stainless sphere (Fig. 1).

Digital images from the fractured pieces of the crowns were used to verify the fragments in all cases final fracture occurred by splitting into two, three and often more parts (Fig. 2).

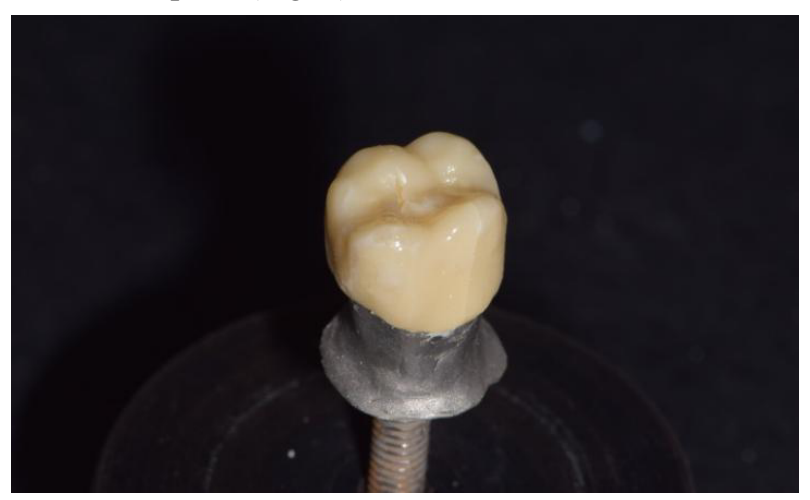

Figure 1. The propagation of cracks in the crown under occlusal loading.

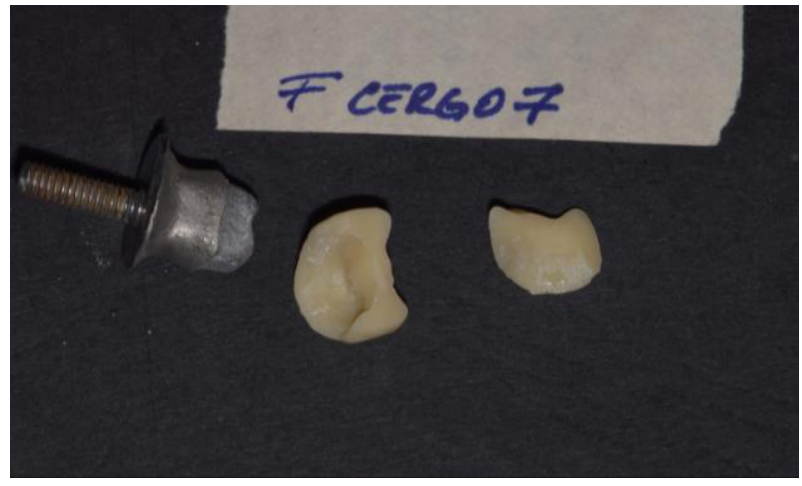

a.

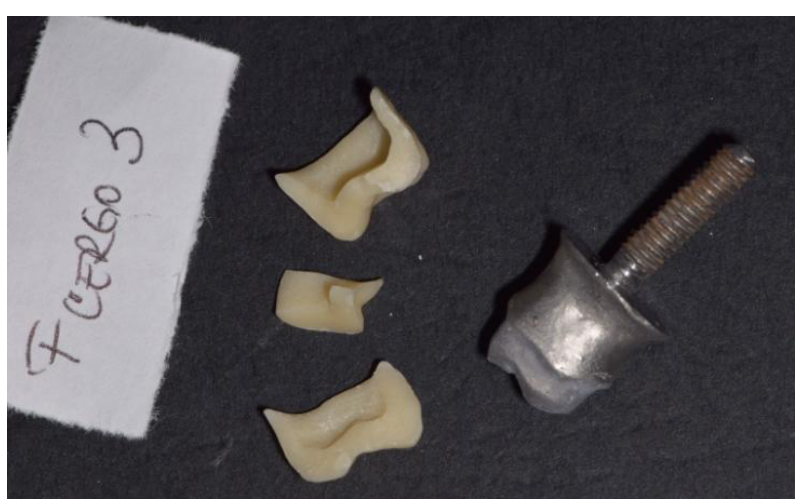

b.

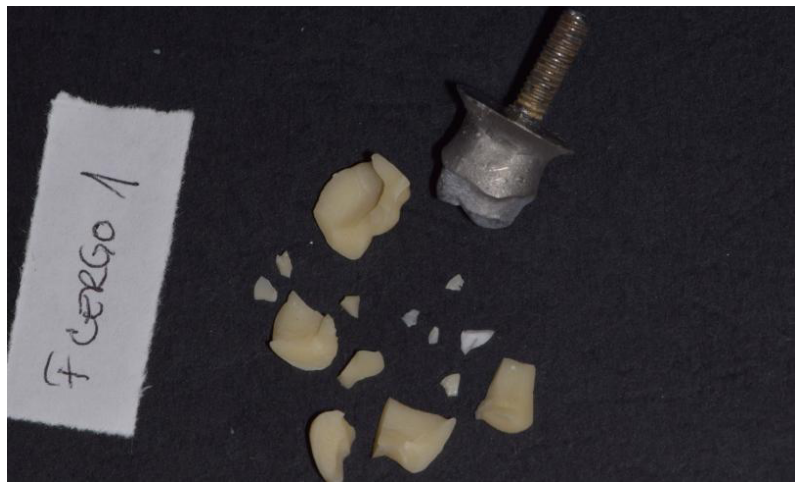

Figure 2. Fracture components of the tested samples: a. sample 7 , sample 3, c. sample 1 . 


\section{Results and discussions}

Experiments show different modes of fracture for the tested samples. The graphically representation of the displacement depending on the load highlights a series of peaks that can be correlated with cracks occurred in crowns. An increased number of peaks are associated with multiple fragments of the fractured samples (Fig. 3).

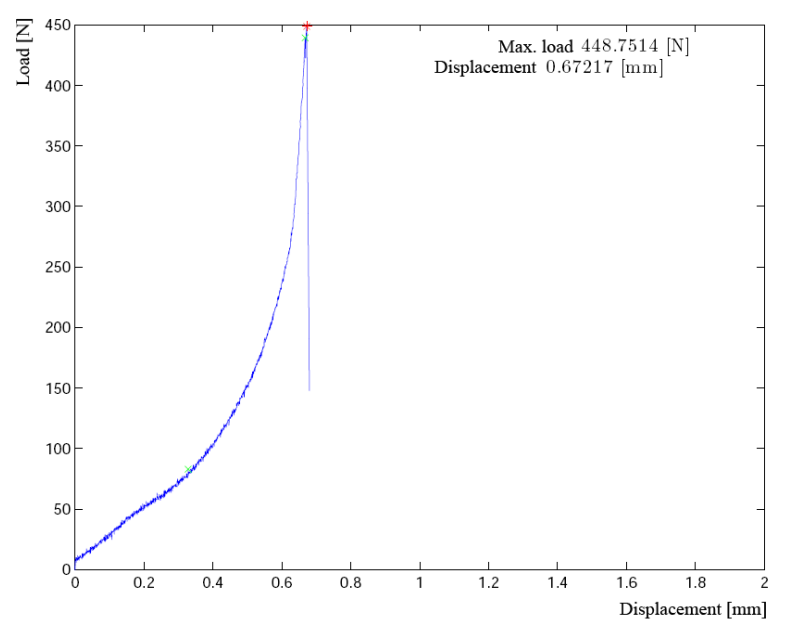

a.

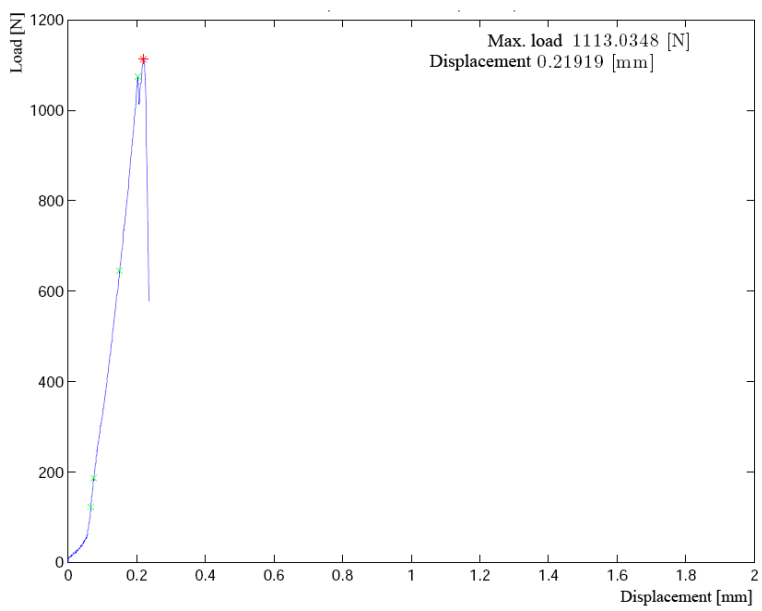

b.

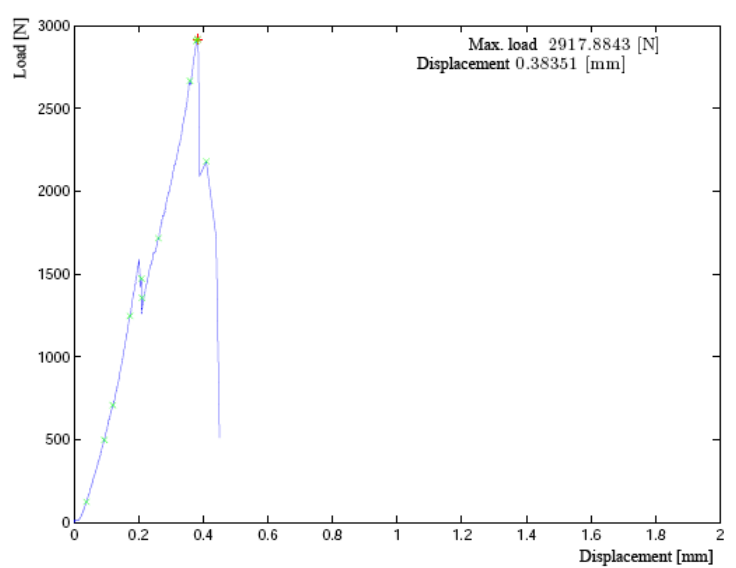

c.

Figure 3. Graphical representation of peaks recorded during mechanical compression tests: a. sample 7, sample 3, sample 1 .

Critical radial cracks initiate from the occlusal contact area with the testing indenter. These cracks ramify and multiple fragments result.
Studies have shown that fracture initiation begins under the indenter tip when a hard spherical antagonist is used for fracture strength testing [14,15].

The mean value of the compression load was 1529.54 $\mathrm{N}$, and of the displacements $0.36 \mathrm{~mm}$ necessary to cause full splits in each monolithic crown system (Fig. 4, 5). Previous mean values of fracture strength in similar studies have varied: $3147 \mathrm{~N}$ for lithium disilicate $(1.5 \mathrm{~mm}$ thickness) [16], $743 \mathrm{~N}$ for 2-mm-thick lithium disilicate [17], $1912 \mathrm{~N}$ for monolithic lithium disilicate $(1.5 \mathrm{~mm}$ thickness) [18], above $1400 \mathrm{~N}$ of 1.5-mm-thick monolithic lithium disilicate crowns [19].

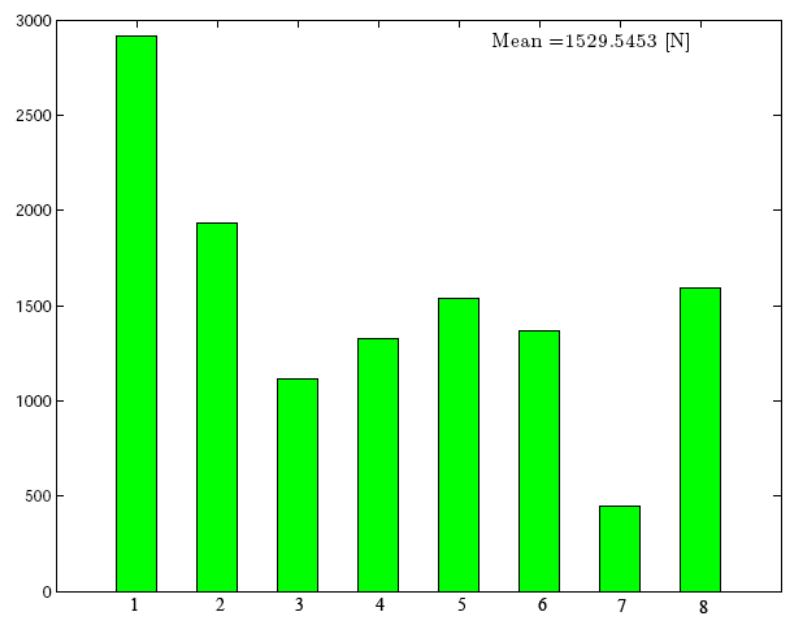

Figure 4. Graphical representation of the maximal compression loads.

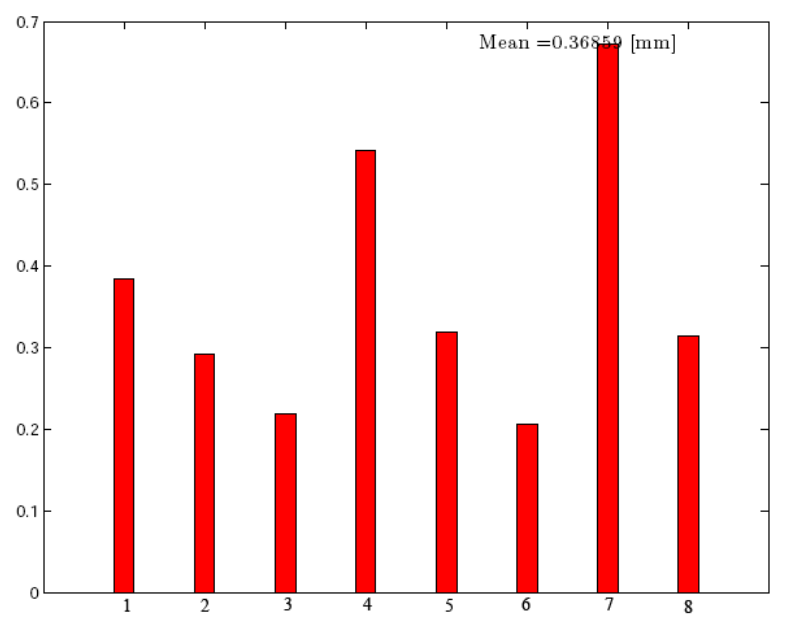

Figure 5. Graphical representation of the maximal displacements.

In order to minimize clinical failure, restorations should be fabricated with consideration of their constituent material properties. The flexural strength of lithium disilicate is $365 \mathrm{MPa}$ [20]. Based on these values, manufacturers recommend tooth reduction for posterior monolithic restorations be $1.5 \mathrm{~mm}$ for lithium disilicate [19]. Different studies evaluated failure of glass ceramic crowns of thicknesses between 1 and $2 \mathrm{~mm}$. Crowns with 2-mm occlusal thickness sustained 1.7 times more cycles and $1.5-\mathrm{mm}$ occlusal thickness, 1.5 times more cycles than 1-mm thick crowns [21]. 
Ceramic materials are known for their relatively high fracture resistance and improved aesthetics, but brittleness remains a concern [22]. Failures are often associated with complete cracking of the crown. Regarding the forces wich lead to failure, it is known that the maximum bite force for adults may reach $700-800 \mathrm{~N}$ [23]. The common occlusal load in oral conditions ranges below $400 \mathrm{~N}$, ceramic crowns rarely undergo rupture due to fracture strength as a result of function in the initial service time. However, rupture could happen due to the accumulation of fatigue stress [24]. Therefore further mechanical analyses simulating the fatigue behavior of this kind of restorations are requires for a more natural reproduction of oral conditions. The stressed area of the material is a key factor for the failure analysis, and numerical simulations may play an important role in the understanding of the behavior of all-ceramic restorations.

Even their many advantages, glass ceramics are brittle materials, and crack initiation and propagation can result in compromise of the restorations. The structural integrity indicated by a significant reduction in fracture strength will be reflected in a poor clinical performance. Therefore the clinical significance of these results is to give an indication of the strength of hot-pressed ceramic crowns to be used in the molar areas as monolithic restorations. Further studies are required to assess the durability of such restorations by different experimental methods, before clinical use [25].

New materials are often introduced into the market and especially in the current practice, without a basic understanding of their clinical performance because long term controlled clinical trials are required, which are both time-consuming and expensive [26].

\section{Conclusion}

Glass ceramics are brittle materials, and crack initiation and propagation can result in compromise of the restorations during functions.

The development of well-designed mechanical experiments could be useful to help to predict clinical survival of these new all-ceramic restorative techniques and materials.

The experiments have to be conducted and interpreted reported to the brittle behavior of ceramic systems.

Because failure is often accompanied by complete cracking of the crowns, preliminary research should represents a compulsory goal.

\section{Acknowledgment}

This work was supported by a grant of the Romanian National Authority for Scientific Research and Innovation CNCS-UEFISCDI, project number PN-II-RUTE-2014-4-0476.
1. F.J. Burke, P.S. Lucarotti, J Dent. 37,12-24, (2009).

2. D.J.Green, Cambridge: University Press; (1998).

3. J.E.Ritter, Dent Mater. 11, 142-6, (1995).

4. H.N. Yoshimura, P.F. Cesar, W.G. Miranda, C.C. Gonzaga, C.Y. Okada and H. Goldenstein, J Am Ceram Soc. 88, 1680-3, (2005).

5. C.C. Gonzaga, P.F. Cesar, W.G. Miranda Jr and H.N. Yoshimura, Dent Mater. 27(4), 394-406, (2011).

6. T. Qasim, M.B. Bush, X. Hu and B.R. Lawn, J Biomed Mater Res B. 73, 179-185, (2005).

7. T. Qasim, C. Ford, M.B. Bush, X. Hu, and B.R. Lawn, J Biomed Mater Res B. 76, 334-339, (2006).

8. G.J.Christensen, J Am Dent Assoc. 130, 409-11, (1999).

9. Y. Zhang, I. Sailer and B.R. Lawn, J Dentistry. 41,1135-47, (2013).

10. Y. Zhang, JJ-W. Lee, R. Srikanth and B R. Lawn, Dent Mater. 29, 1201-8, (2013).

11. G.J.Christens, JAm Dent Assoc. 145, 192-4, (2014).

12. Y. Zhang, I. Sailer, B.R..Lawn, J Dent. 41(12):113547 (2013).

13. J.R..Kelly, Dental Clinics of North America. 48:513530 (2004).

14. E.D. Rekow, G. Zhang, V. Thompson, J.W. Kim, P. Coehlo and Y. Zhang, J Biomed Mater Res B Appl Biomater. 88, 436-46, (2009).

15. J.O. Clausen, M. Abou Tara, M. Kern, Dent Mater 26:533-8 (2010).

16. K. Nakamura, A. Harada, R. Inagaki, T. Kanno, Y. Niwano, P. Milleding, U.Örtengren, Acta Odontol Scand. 1, 1-7, (2015).

17. M. Dhima, D.A. Assad, J.E. Volz, K.N. An, L.J. Berglund, A.B. Carr and T.J.Salinas, J Prosthodont. 22, 256-60, (2013).

18. T. Sun, S. Zhou, R. Lai, R. Liu, S. Ma, Z. Zhou, S. Longquan, J Mech Behav Biomed Mater. 35, 93-101, (2014).

19. B. Baladhandayutham, N.C. Lawson, J.O. Burgess, J Prosthet Dent. 114(2), 266-71, (2015).

20. W.S. Lin, C. Ercoli, C. Feng, D.Morton, 21, 353-62, (2012).

21. M. Dhima, A.B. Carr, T.J. Salinas, C. Lohse, L. Berglund, K.A. Nan, J Prosthodont. 23, 353-7, (2014).

22. Y. Zhang, Z. Mai, A. Barani, M. Bush, B.Lawn, Dent Mater. 32(3), 442-9, (2016).

23. K. Nishigawa, E. Bando, M. Nakano, J Oral Rehabil 28, 485-491, (2001).

24. O. El-Mowafy, J.F. Brochu, "Longevity and clinical performance of IPSEmpress ceramic restorations - a literature review," J Canad Dent Assoc B, 233-237, (2002).

25. L. Porojan, S. Porojan, A.Cernescu, C. Savencu, Int J Syst Appl, Eng \& Dev, 10, 266-269, (2016).

26. S. Nasrin, N. Katsube, R.R. Seghi and S.I. Rokhlin, J Biomech. 10, pii:S0021-9290(16)30550-4, (2016).

\section{References}

